\title{
EPR Spectra of Multispin Systems. Application to Poly-m-p-Aniline
}

\author{
P. Juszyński ${ }^{a}$, A. Twardowski ${ }^{a}$, J. Gosk $^{a, b}$, I. Kulszewicz-BAJer ${ }^{c}$, M. PAWŁowski $^{d}$, \\ K. DZIATKOWSKI ${ }^{a}$ AND J. SzCZYTKO ${ }^{a}$ \\ ${ }^{a}$ Institute of Experimental Physics, Faculty of Physics, University of Warsaw, Hoża 69, 00-681 Warsaw, Poland \\ ${ }^{b}$ Faculty of Physics, Warsaw University of Technology, Koszykowa 75, 00-662 Warsaw, Poland \\ ${ }^{c}$ Faculty of Chemistry, Warsaw University of Technology, Noakowskiego 3, 00-664 Warsaw, Poland \\ ${ }^{d}$ Institute of Electronic Materials Technology, Wólczyńska 133, 01-919 Warsaw, Poland
}

In this communication we present NiceMagnetic, software for simulations and analysis of EPR spectrum and SQUID measurements. Systems of arbitrary number $N$ of spin-only magnetic moments $(S \neq 0$ and $L=0)$ were considered within the lines of the Heisenberg and Zeeman Hamiltonians. Effective algorithms used for the computations are discussed. This includes modified secant method adapted to calculate resonance fields with demanded precision. Finally, application of NiceMagnetic to experimental SQUID magnetization and EPR spectra of poly- $m$ - $p$-aniline, one of recently synthesized high spin polymers containing unpaired electrons will be presented. As a result, exchange integral between electron and nitrogen nuclei spins (ligand hyperfine interaction) $J=6.3 \times 10^{-4} \mathrm{~cm}^{-1}$ was estimated.

PACS: 02.70.-c, 75.10.-b

\section{Introduction}

Recently considerable attention has been paid to magnetic materials, which are hoped for application in spintronics. Progress in such studies depends both on new experimental data as well as comprehensive analysis of the data. This includes development of software able to handle large spin systems. Often used techniques are EPR and SQUID magnetometry.

There are some softwares prepared for such data analyzes (XSophe [1], ZFSFIT [2], EasySpin [3]) but they either use noneffective algorithms or do not guarantee demanded precision.

Moreover, we want to analyze both EPR and SQUID experimental data in the same consistent way.

In this communication we present such software (NiceMagnetic module) capable to describe arbitrary number of magnetic moments simultaneously calculating EPR spectra and magnetization signal.

\section{Model - used method}

Systems of arbitrary number $N$ of spin-only magnetic moments $(S \neq 0$ and $L=0)$ were considered within the lines of the Heisenberg and Zeeman Hamiltonians [4]:

$$
H=B \sum_{i=1}^{N} \beta_{i} g_{i} \hat{S}_{i}^{z}-\sum_{i=1}^{N} \sum_{j>i}^{N} \hat{S}_{i} J_{i j} \hat{S}_{j} .
$$

Here $B$ is the magnetic field, $\beta_{i}$ is the Bohr magneton or nucleus magneton for electron or nucleus respectively, $g_{i}$ is the $g$-factor, $S_{i}$ is the spin operator of particle $i$ and $J_{i j}$ is exchange integral between $i$ and $j$ particle.

The full Hamiltonian of the considered system can be numerically solved returning energy spectrum $E_{i}(B)$ and eigenstates as a function of magnetic field for chosen exchange interaction between spins. Finding positions of resonance fields (for which energy between two different eigenvalues is equal to $\Delta_{i j}(B)=E_{i}(B)-E_{j}(B)=\hbar \omega$ seen in EPR is a numeric challenge. It can be solved in some different ways.

Even though the adaptive method used by Stoll [5] in Easyspin is fast, it cannot return resonant fields in demanded precision (although estimates it).

To assure precision ZFSFIT [2] uses bisection method (searching $x$-intercept of the function $f(x)=\Delta_{i j}(x)-$ $\hbar \omega)$ for all pairs of eigenstates separates. It is why this technique is slower [1]. We used a modification of the scan method instead of bisection so our algorithm is faster and still calculates resonance fields with demanded precision.

\section{Example of using - application to poly- $m$ - $p$-aniline}

Application of NiceMagnetic to experimental magnetization and EPR spectra of poly- $m$ - $p$-aniline, one of the recently synthesized high spin polymers containing unpaired electrons is presented.

High spin materials are chemical molecules containing unpaired electrons in which ground state is a high spin 
state [6] (high spin state results from coupled electronics spins $\left.S_{i}=1 / 2\right)$.

These systems are important from the point of view of a new interesting physics (ferromagnetic coupling mechanism) and its application (for example new kind of magnets).

Poly- $m$ - $p$-aniline [7] is one of the recently synthesized high-spin polymers containing unpaired electrons. It gives hope to combine advantages of high-spin systems and polymers in one material.

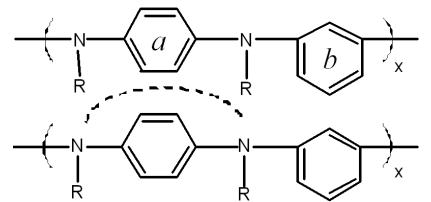

Fig. 1. Chemical formula of poly- $m$ - $p$-aniline before (upper part) and after (lower part) oxidation.

Chemical formula of poly- $m$-p-aniline is shown in Fig. 1. Poly- $m$ - $p$-aniline contains two kinds of benzene-like cycles (marked as $a$ and $b$ in Fig. 1). By chemical oxidation unpaired electron appears (represented by the dashed line). Each unpaired electron is localized around only two nitrogen nuclei since $a$ cycle enables move of an electron along polymer chain and $b$ cycle does not.

It is why we take into account only electron and two nitrogen nuclei spins influence on it as a model system to describe poly- $m$ - $p$-aniline.

\section{Data analysis details}

EPR spectrum of poly- $m$ - $p$-aniline and magnetization for various temperatures was measured.

NiceMagnetic returns magnetization signals (as a function of $B$ and temperature as a parameter).

NiceMagnetic returns not an EPR spectrum, but only positions of the resonance fields $B_{k}$ (see Fig. 2). EPR signal is modeled by sum of derivatives of the Gauss profiles (the Lorentz was also tested)

$$
f(B)=a_{0}+\sum_{k=1}^{N} a_{k} \frac{B-B_{k}}{\sigma_{k}} \exp \left(\frac{-\left(B-B_{k}\right)^{2}}{2 \sigma_{k}^{2}}\right),
$$

where $a_{k}$ are multiplicative constants, and $\sigma_{k}$ are parameters responsible for width of the "peaks".

Second integral of the peaks is equal to so-called intensities of the transition $I_{k}$ (also calculated by NiceMagnetic) between states $u_{i}$ and $u_{j}$ with energies $E_{i}$ and $E_{j}$ respectively, given by

$$
\begin{aligned}
I_{k} & \propto\left|\left\langle u_{i}\left|\hat{M}^{x}\right| u_{j}\right\rangle\right|^{2} \\
& \times \frac{\exp \left(-E_{i} / k_{\mathrm{B}} T\right)-\exp \left(-E_{j} / k_{\mathrm{B}} T\right)}{\sum_{q} \exp \left(-E_{q} / k_{\mathrm{B}} T\right)} .
\end{aligned}
$$

Here $M^{x}$ is the operator of projection of the magnetic moment on $x$ axis.

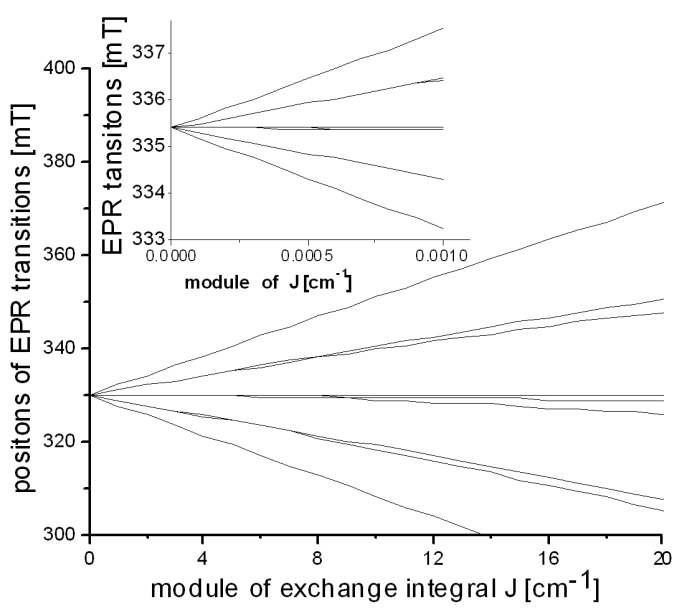

Fig. 2. Positions of resonance fields in poly- $m-p$ -aniline problem as a function of exchange integral $J$ in a broad range (calculated by NiceMagnetic). Inset: Positions of resonance fields for values of $J$ characteristic for poly- $m$ - $p$-aniline.

Equation (3) gives now form

$$
f(B)=a_{0}+a_{1} \sum_{k=1}^{N} I_{k} \frac{\left(B-B_{k}\right)^{2}}{\sigma_{k}^{3}} \exp \left(\frac{-\left(B-B_{k}\right)^{2}}{2 \sigma_{k}^{2}}\right) .
$$

The width parameters $\sigma_{k}$ are not calculated by NiceMagnetic because they are external parameters in the discussed model.

Taking $\sigma_{k}$ equal for all transitions does not provide a reasonable agreement with the experimental data.

The upper part of Fig. 3 shows five characteristic peaks. This means that we are in low $J$ regime (see Fig. 2). Nine resonance fields divided into five groups are presented.

The same $\sigma_{k}$ for each transition into one group was taken in further analysis. Fitting procedure with $J, a_{0}$, $a_{1}$ and five different $\sigma_{k}$ was performed. A very good quantitative agreement of this model with experimental data (see upper part in Fig. 3) was found.

We note that fitting of EPR spectra and magnetization data was made simultaneously.

As a result, exchange integral between electron and nitrogen nucleus was estimated as $J=6.3 \times 10^{-4} \mathrm{~cm}^{-1}$.

Different broadening for different group can result from anisotropy. $J$ can be different for different directions, so peaks in result spectrum is the sum of many peaks with different $J$. In a polymer, while interaction between nitrogen and electron is not isotropic, the spectrum is averaged over all possible directions of the magnetic field. As one can see in Fig. 2, the more outlying from central resonance field the peak is, the most its position varies with $J$. It is why outermost peaks will have the largest $\sigma_{k}$. This is equivalent to fitting results. 

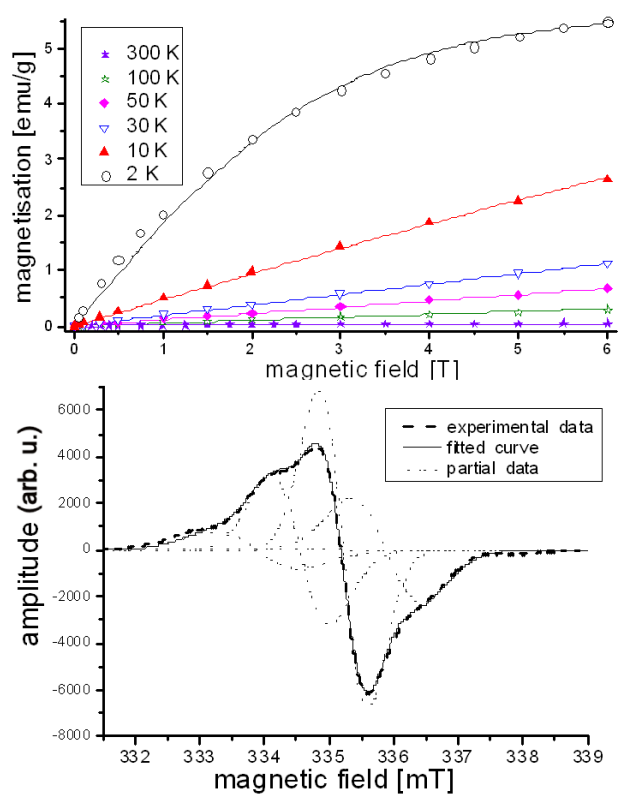

Fig. 3. Experimental results with fitting curves. (a) Magnetization as a function of external magnetic field for various temperatures. Fitted curved - lines, experimental results - points. (b) EPR spectrum: experimental data (solid line), fitted curve (dashed line very close to experimental curve), parts of fitted curve -5 peaks for 5 groups discussed in text (pointed line); fifth peak (for the highest magnetic field) is so broad that it is hardly visible.

\section{Summary and discussion}

Software appropriate for EPR spectra and magnetization analysis was presented. Calculating resonance fields, crucial for some kinds of experiments, is possible. Proposed model of the Zeeman and Heisenberg Hamilto- nians to explain experimental data for high-spin polymer provides a reasonable description. Exchange integral $J=6.3 \times 10^{-4} \mathrm{~cm}^{-1}$ for interaction between nitrogen and electron in poly- $m$ - $p$-aniline was obtained.

Further improvements of the procedure can be proposed:

Parallel calculation can shorten calculation time.

One can notice that diagonalised matrix can be separated into some smaller matrixes (one matrix per one projection of the magnetic moment on $x$ axis). The total length of time of diagonalizations of smaller matrixes is much more shorter than the time necessary to diagonalize the big one.

The presented method can be useful in many various physical situations, in particular to analyze multispin systems of magnetic impurities in semiconductors or clusters of magnetic ions.

\section{References}

[1] G.R. Hanson, J. Inorg. Biochem. 98, 903 (2004).

[2] G. Morin, D. Bonnin, J. Magn. Reson. 136, 176 (1999).

[3] S. Stoll, A. Schweiger, J. Magn. Reson. 178, 42 (2006).

[4] A. Abragam, B. Bleaney, Electron Paramagnetic Resonance of Transition Ions, Clarendon Press, Oxford 1970 .

[5] S. Stoll, A. Schweiger, Chem. Phys. Lett. 380, 464 (2003).

[6] M.M. Wienk, R.A.J. Janssen, J. Am. Chem. Soc. 119, 4492 (1997).

[7] I. Kulszewicz-Bajer, M. Zagórska, I. Wielgus, M. Pawłowski, J. Gosk, A. Twardowski, J. Phys. Chem. B 111, 32 (2007). 